\title{
Uso de la función de arrastre para generar experiencias de aprendizaje de la demostración en geometría
}

Artículo recibido: 30-05-2010 | Artículo aprobado:02-07-2010

Use of Dragging Function to Generate Demonstration Geometry Learning Experiences

\section{Leonor Camargo*}

Patricia Perry**

Carmen Samper**

Óscar Molina****

Armando Echeverry

Resumen: En este artículo reportamos un uso novedoso de la función de arrastre de los programas de geometría dinámica, puesto al servicio de la enseñanza de la demostración. Éste sucede cuando el profesor, atento a enriquecer la actividad matemática de sus estudiantes, aprovecha el recurso tecnológico, disponible en el aula, para sugerir una vía de validación de la conjetura de un grupo de estudiantes e impulsa con ello una experiencia significativa de participación de los miembros de la clase en la actividad demostrativa. ${ }^{1}$

Palabras clave: geometría dinámica, función de arrastre, aprendizaje, actividad demostrativa, papel del profesor.

\begin{abstract}
This article reports a novel use of dragging function to dynamic geometry programs for teaching demonstration. This takes place when the teacher willing to enriching students' mathematical activity, takes advantage of the technological resource available in class to suggest a way for validating the conjecture of a group of students, and promotes a meaningful experience of participation in the proving class members.
\end{abstract}

Keywords: Dynamic Geometry, Dragging Function, Learning, Proving Activity, Teacher's Role.

\footnotetext{
* Profesora asociada Universidad Pedagógica Nacional, Icamargo@pedagogica.edu.co

* Investigadora Universidad Pedagógica Nacional, pperryc@yahoo.com.mx

*** Profesora titular Universidad Pedagógica Nacional, csamper@pedagogica.edu.co

**** Profesor asistente Universidad Pedagógica Nacional, ojmolina@pedagogica.edu.co

***** Profesor ocasional Universidad Pedagógica Nacional, aecheverri@pedagogica.edu.co

1 Los cinco autores de este artículo son responsables de la línea Aprendizaje y Enseñanza de la Geometría del grupo Didáctica de la Matemática del Departamento de Matemáticas de la Universidad Pedagógica Nacional.

2 Agradecemos el apoyo económico brindado por el Centro de Investigaciones de la Universidad Pedagógica Nacional (CIUP) para el desarrollo de la investigación que nos proporcionó los datos de la presente comunicación.
} 
Uso de la función de arrastre para generar experiencias de aprendizaje de la demostración en geometría Leonor Camargo | Patricia Perry | Carmen Samper | Óscar Molina | Armando Echeverry

\section{Introducción}

Nuestro propósito en este artículo es reportar una experiencia de aula en la que surgió, de manera no prevista, un uso novedoso de la función de arrastre de los programas de geometría dinámica, uso que organizó la actividad colectiva de la clase, estimuló la actividad demostrativa de los estudiantes y favoreció su participación en la construcción de conocimiento. Sucedió en el marco de un experimento de enseñanza (Cobb, 2000), realizado en un curso de geometría plana de nivel universitario. El experimento tenía como objetivo identificar la evolución de la participación de los estudiantes en la producción y justificación de enunciados para conformar una porción de un sistema axiomático.

Consideramos que este reporte contribuye a atender la solicitud formulada por Herbst (2002) sobre la necesidad de idear formas de organización de las clases que favorezcan la participación de los estudiantes en la actividad de demostrar, en los diferentes niveles educativos. La revisión de la literatura nos permite afirmar que los estudios adelantados sobre la función de arrastre en la enseñanza y el aprendizaje de la geometría se han centrado en cómo la usan los estudiantes cuando resuelven problemas (Olivero, 1999; Arzarello, Olivero, Paola y Robutti, 2002) pero no se ha explorado suficientemente su potencial como organizador de la actividad colectiva en clase.

La experiencia particular tomó forma a partir de la resolución de un problema, por parte de los estudiantes, en el que se requiere explorar una situación en un entorno de geometría dinámica (Cabri). El problema hace parte de un conjunto de tareas implementadas durante el semestre académico, a través de las cuales los estudiantes se involucran en la construcción de una porción de sistema axiomático para la geometría plana al analizar situaciones cuya exploración en Cabri conduce a la formulación e interpretación de conjeturas y luego justificar dichas conjeturas. Antes de referirnos a la situación particular, en primer lugar, damos cuenta de algunas ideas que fundamentan la planeación de la clase en la que sucedió la experiencia que aquí nos ocupa. En segundo lugar, contextualizamos la experiencia dentro del marco investigativo del cual hace parte. En tercer lugar, describimos, interpretamos y analizamos los sucesos de la clase. Finalmente extraemos algunas conclusiones.

\section{Consideraciones teóricas}

Desde nuestro punto de vista, el constructo actividad demostrativa incluye dos procesos conformados por acciones que sustentan la producción de una conjetura y su demostración. Estas acciones consisten en: la exploración de una situación para buscar regularidades, la formulación de conjeturas con base en las regularidades encontradas, la respectiva constatación de la veracidad del hecho geométrico enunciado, y la búsqueda y organización de ideas que conforman la demostración. Ésta se refiere a un argumento, generalmente de índole deductivo, fundamentado en un sistema axiomático de referencia del que puede hacer parte el enunciado demostrado. En ese sentido, coincidimos con investigadores (Hanna, 2000; Mariotti, 2006) que consideran la demostración como una actividad primordial en la enseñanza de las matemáticas, mediante la cual se gana comprensión sobre los enunciados en juego y éstos se organizan con el objeto de validarlos al interior de sistemas teóricos. Dado que los principios y las reglas que rigen la producción del discurso son establecidos por un grupo humano específico, éstos pueden 
variar en el tipo de argumentación aceptado - aunque se espera una evolución desde argumentaciones informales hasta unas deductivas; por lo anterior, decimos que la actividad demostrativa es de carácter sociocultural, condicionada por el contexto en donde se lleva a cabo y por el dominio específico al interior del cual se está actuando (Alibert y Thomas, 1991; Hoyles, 1997; Radford, 1994; Godino y Recio, 2001; Mariotti, 2006).

En consonancia con nuestro concepto de actividad demostrativa, concebimos su aprendizaje como un proceso sociocultural en el que los aprendices se apropian y usan convenciones comunicativas cada vez más próximas a las usadas por la comunidad cultural de referencia. Vemos la clase de matemáticas como una comunidad de práctica (Wenger, 1998) en la que el profesor, miembro experto de ésta, es el responsable de ofrecer recursos para estructurar la actividad de los estudiantes y de aprovechar situaciones de aula como oportunidades de aprendizaje. Por su parte, los estudiantes, novatos en la comunidad, tienen la oportunidad de aprender a demostrar al comprometerse con un repertorio de prácticas propias de la actividad demostrativa. A medida que participan de estas prácticas, desarrollan ideas de lo que significa demostrar y de cómo pueden ser partícipes legítimos de la producción de demostraciones.

Indudablemente, el repertorio de prácticas está condicionado por los medios con los que cuenta la comunidad de la clase para llevar a cabo la tarea que se propone, y por las pautas que se van negociando para el uso de los mismos. En particular, cuando se dispone de un programa de geometría dinámica, el uso de funciones como el arrastre, se vuelve un aspecto característico de las prácticas de la comunidad. Eventualmente, puede surgir un uso atípico de alguna fun- ción, que posteriormente se evalúa como favorable para la práctica que se está llevando a cabo y que se vislumbra puede ser una forma útil para sacar provecho de los programas de geometría dinámica. Es el caso del uso de la función de arrastre que queremos destacar en este artículo.

\section{Contexto de la experiencia}

El experimento de enseñanza se llevó a cabo con 21 estudiantes inscritos en un curso de geometría plana del programa de Licenciatura en Matemáticas de la Universidad Pedagógica Nacional (Bogotá, Colombia). A lo largo del semestre, los estudiantes (17 - 20 años) participaron en la construcción colectiva de una porción de un sistema axiomático para la geometría euclidiana plana relacionado con propiedades de ángulos, triángulos y cuadriláteros. Además, trabajaron en la resolución de problemas abiertos y ganaron conocimientos sobre el programa de geometría dinámica Cabri, para usarlo con cierta destreza en la exploración de figuras y la verificación de conjeturas producto de la exploración. La experiencia particular en la que tuvo lugar el hallazgo investigativo que reportamos en este artículo se realizó hacia el final del curso con una duración de cuatro horas, distribuidas en dos sesiones de clase. El reporte está basado en la trascripción de las grabaciones de audio y video de las sesiones de clase, complementada con notas de campo que tomó uno de los miembros del equipo de investigación que estuvo presente como observador no participante.

Consideramos que la experiencia ejemplifica lo que puede pasar en una clase de geometría de nivel universitario cuando se implementan las ideas teóricas expuestas en la sección previa, que fundamentan nuestra aproximación pedagó- 
Uso de la función de arrastre para generar experiencias de aprendizaje de la demostración en geometría Leonor Camargo | Patricia Perry | Carmen Samper | Óscar Molina | Armando Echeverry

gica. La participación de los estudiantes, trabajando en parejas, comienza con la resolución de un problema que da lugar a la formulación de diversas conjeturas sobre las propiedades geométricas que debe tener un punto para cumplir una condición impuesta en el enunciado. La certeza de las conjeturas que se formulan se logra usando funciones del programa de geometría dinámica, tales cono el arrastre y la medida; pero la validación de éstas requiere, según las normas establecidas en clase, el uso de enunciados que conforman el sistema axiomático que se ha construido hasta el momento. En esta experiencia, dos de las conjeturas referidas a la construcción del punto se salían del marco de propiedades validables a través de la teoría disponible, razón por la cual la profesora se refirió a ellas como 'problemáticas'. Pero, en lugar de descartarlas o dejarlas de lado decidió, sobre la marcha, hacer uso del arrastre para encontrar una vía mediante la cual hacer la validación. Así, logró que la producción de los estudiantes, aunque inesperada, fuera útil para avanzar en la construcción de conocimiento matemático en la clase.

\section{La experiencia}

En la primera sesión, los estudiantes recibieron el enunciado del problema escrito en una hoja, sin alguna figura de referencia. Éste decía:

Se da una recta $m$ y dos puntos $P$ y $Q$ en el mismo semiplano determinado por $m$. Determinen el punto $R$ de $m$ para el cual la suma de las distancias $P R+R Q$ es la menor. a) Describan el procedimiento geométrico y la exploración hecha para encontrar la posición de $R$. b) Formulen una conjetura. c) Escriban los pasos principales de la demostración de la conjetura.
Los estudiantes se distribuyeron en nueve parejas y un trío. Los grupos hicieron en Cabri la construcción de una recta $m$, ubicaron dos puntos $P$ y $Q$ en uno de los semiplanos y un punto $R$ sobre la recta; trazaron los segmentos $P R$ y $Q R$, encontraron la medida de sus longitudes y establecieron la suma de $P R$ y $Q R$ (Figura 1). Algunos impusieron condiciones especiales a $P$ y $Q$, como estar a la misma distancia de $m$ o ambos sobre una recta perpendicular a $m$.

A continuación, comenzaron el proceso de exploración haciendo uso del arrastre sobre objeto (Arzarello, Olivero, Paola y Robutti, 2002), es decir, moviendo el punto $R$ sobre la recta $m$ con el objeto de encontrar la característica de éste que se pedía. En busca de la única posición de $R$ para obtener la suma mínima, algunas parejas obtuvieron aparentemente el mismo resultado para varios puntos de la recta, hecho que los llevó a aumentar el número de cifras decimales para afinar el resultado de la suma. Por esta vía, ubicaron al punto $R$ en la posición que parecía cumplir la condición de la suma mínima.

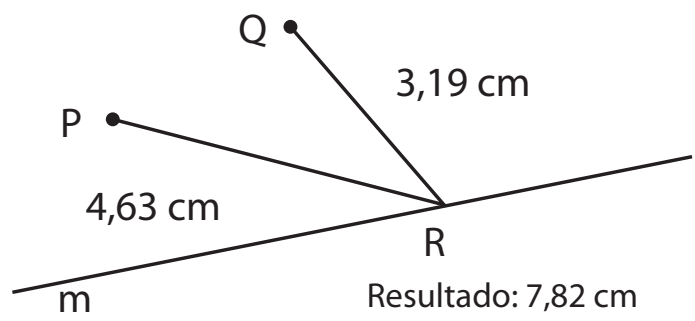

Figura 1

Una vez asegurada la existencia de dicho punto, los estudiantes empezaron a buscar propiedades geométricas para caracterizar la posición $R$ y formular una conjetura al respecto, lo que los llevó a hacer construcciones auxiliares, tomar otras medidas, hacer 
un arrastre 'errante' (Arzarello, Olivero, Paola y Robutti, 2002) de los puntos $P$ y $Q$, en busca de configuraciones especiales o regularidades en la figura. La profesora iba desplazándose por cada grupo para interactuar con los estudiantes y enterarse de la exploración realizada, la conjetura formulada y los avances en el proceso de su demostración. Vea- mos, a manera de ejemplo, un fragmento de la conversación que sostuvo con el grupo conformado por Daniel, Orlando y Melisa en la que se observa que el uso del arrastre es admitido por profesora y estudiantes como recurso para verificar que la conjetura era admisible. La representación sobre la que conversan es similar a la Figura 2.

Figura 2

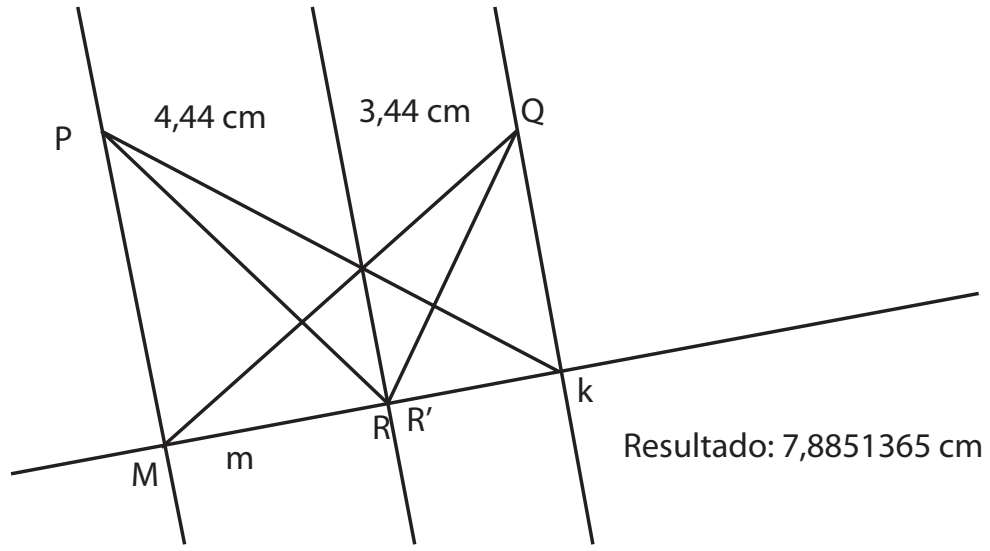

\begin{tabular}{|l|l|}
\hline Profesora: & {$[\ldots]$ han hecho cosas interesantes. Explícame [se dirige a Melisa]. } \\
\hline Melisa: & Tomamos dos puntos $P$ y $Q$ cualquiera. Trazamos las perpendiculares a/ ${ }^{3}$ \\
\hline Profesora: & /pero... ¿comenzaron así? O escogieron primero un [punto] $R$. \\
\hline Daniel: & $\begin{array}{l}\text { Escogimos un [punto] } R \text { y comenzamos a moverlo, a ver dónde [la suma mínima] } \\
\text { era la más chiquita. }\end{array}$ \\
\hline Profesora: & $\begin{array}{l}\text { Sí. Entonces después ¿qué?... después fue cuando construyeron las } \\
\text { perpendiculares, porque cuando yo pasé por aquí [minutos antes] no las tenían. }\end{array}$ \\
\hline Melisa: & $\begin{array}{l}\text { Sí profe. Y entonces, los puntos de intersección [con } m \text { ] los llamamos } M \text { y } K \text {, ¿sí? } \\
\text { Trazamos el segmento } M Q \text { y el segmento } P K . \text { Y entonces se intersecan. }\end{array}$ \\
\hline Melisa: & $\begin{array}{l}\text { Tr...] } \\
M Q \text { y } P K], \text { y sí nos da. Y ya... comenzamos a mover. Lo que pasa es que el punto de } \\
\text { intersección [con la recta } m \text { ] lo llamamos } R^{\prime} \text { y entonces empezamos a mover un } R \\
\text { cualquiera y comenzamos a mover las distancias y entonces sí nos da. }\end{array}$ \\
\hline Profesora: & $\begin{array}{l}\text { Hay que escribir esa conjetura. Está muy interesante }{ }^{3} . \\
{[\ldots]}\end{array}$ \\
\hline Profesora: & $\begin{array}{l}\text { O sea, si los mueves... [Mueven a } P \text { y } Q \text { ]. Sin importar [la posición de] } P \text { y } Q . Y \text { ahora } \\
\text { mueve a } R \text { para ver la posición... mueve. Parece que sí [funciona la construcción]. }\end{array}$ \\
\hline
\end{tabular}

3 Usamos el signo / para indicar que la persona que está hablando es interrumpida por el siguiente participante de la conversación y por eso la frase no está completa.

4 Los estudiantes debían redactar como conjetura una proposición condicional en la que el antecedente fuera la caracterización geométrica del punto $\mathrm{R}$ y el consecuente fuera que la suma $\mathrm{PR}+\mathrm{RQ}$ es la menor. 
Uso de la función de arrastre para generar experiencias de aprendizaje de la demostración en geometría Leonor Camargo | Patricia Perry | Carmen Samper | Óscar Molina | Armando Echeverry

El arrastre se usa en dos momentos de la exploración. Primero, como lo explica Daniel, para encontrar una posible ubicación del punto $R$. Segundo, para verificar que la construcción auxiliar hecha sí produce el punto solicitado; esta idea es expresada por la profesora en la última intervención. A través de conversaciones como la anteriormente transcrita, la profesora se enteró de las diferentes conjeturas.

Después del trabajo en grupos, algunos de éstos presentaron públicamente sus conjeturas, mostrando la representación gráfica correspondiente. Por medio del arrastre, entre todos admitieron seis de estas conjeturas. Con la presentación de las conjeturas se culminó la primera sesión de clase. La profesora pidió a los estudiantes pensar en la demostración de su respectiva conjetura, pues ningún grupo había logrado hacerlo durante el trabajo en parejas. Ella les sugirió tomar otro punto sobre la recta $m$ y comparar la suma de las distancias a $P$ y $Q$.
Como preparación para la segunda sesión, la profesora registró en acetatos las figuras correspondientes a las seis conjeturas y la síntesis de cada una de ellas según había sido descrita por los grupos (Figura 3). En clase proyectó las figuras en el tablero y se refirió a ellas: señaló las conjeturas (i) y (ii) como posibles soluciones a un problema que se puede considerar como la "simplificación" del original, puesto que al imponer condiciones adicionales a los puntos $P$ y $Q$ las conjeturas se convertían en casos particulares, más sencillos, de la situación general. Evaluó positivamente el esfuerzo de enfrentarse al problema mirando opciones más simples y dejó para más adelante la demostración de esas conjeturas. Después invitó a revisar las conjeturas (iii) y (iv), que denominó 'problemáticas'. Indicó que las conjeturas aludían a la congruencia de ángulos, pero que no proponían una vía de construcción para el punto $R$ justificable con la teoría disponible; si bien daban cuenta de una propiedad geométrica que podía constatarse al tener construido $R$, no informaban cómo construir el punto, a diferencia de las conjeturas (v) y (vi).

\begin{tabular}{|l|l|l|}
\hline $\begin{array}{l}\text { (i) Caso Particular: si } P \text { y } Q \text { están } \\
\text { sobre una perpendicular a la } \\
\text { recta } m \text { y } R \text { es colineal a } P \text { y } Q \\
\text { entonces } P R+R Q \text { es la suma } \\
\text { mínima. }\end{array}$ & $\begin{array}{l}\text { (ii) Caso Particular: } \operatorname{Si} P \text { y } Q \\
\text { equidistan de } m \text { y } R \text { es el punto } \\
\text { medio del segmento } A B \text {, entonces } \\
P R+R Q \text { es la suma mínima. }\end{array}$ & $\begin{array}{l}\text { (iii) Si los ángulos 1 y } 2 \text { son } \\
\text { congruentes entonces } P R+R Q \text { es la } \\
\text { suma mínima. }\end{array}$ \\
\hline
\end{tabular}




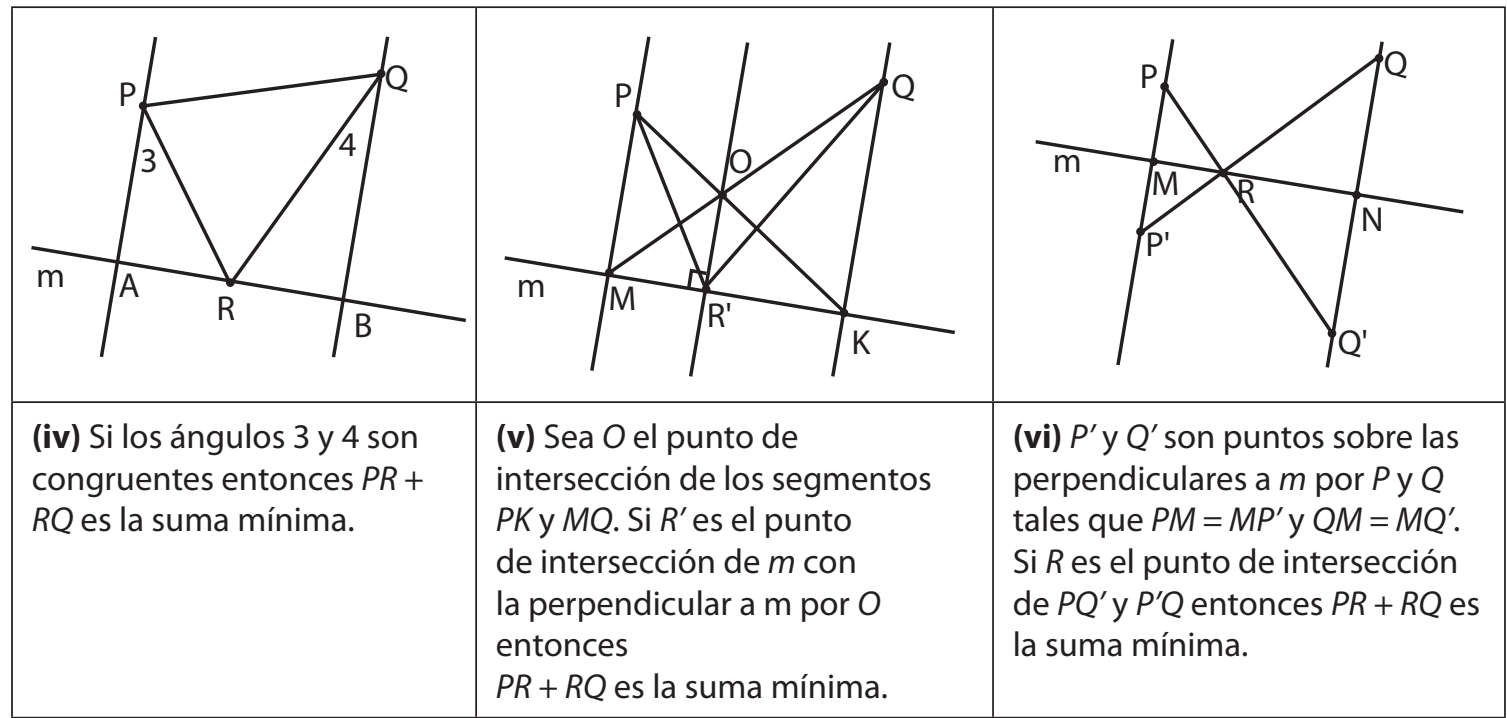

Figura 2

A continuación, solicitó que algún estudiante pasara al tablero a presentar el avance logrado en la demostración de su respectiva conjetura. Darío se ofreció a demostrar la conjetura (vi). El siguiente extracto de la trascripción nos muestra la interacción pública del estudiante con la profesora, en la que él expone el bosquejo de la demostración. Éste se constituyó en la base para la validación de las demás conjeturas, incluso de las problemáticas.

\begin{tabular}{|l|l|}
\hline Darío: & $\begin{array}{l}\text { Bueno, pues primero, para esta demostración yo me di cuenta que con uno } \\
\text { de estos [segmentos] bastaría }\left[P Q^{\prime} \text { o } Q P^{\prime} ; \text { dado que ellos se cortan en } m ;\right. \\
\text { Figura 3 (vi)], entonces podemos quitar una... }\end{array}$ \\
\hline Profesora: & $\begin{array}{l}\text { Sí, con uno de ellos bastaría. Si señor... quitemos ese [señala en el acetato } \\
\left.\text { el segmento } P Q^{\prime}\right] \ldots \text { sí. Hagamos el dibujo como propones. }\end{array}$ \\
\hline Darío: & $\begin{array}{l}{\left[\text { Hace una figura en el tablero dejando sólo } P^{\prime} Q ; \text { la representación luce }\right.} \\
\text { como la Figura } 4(\mathrm{a})] .\end{array}$ \\
\hline
\end{tabular}

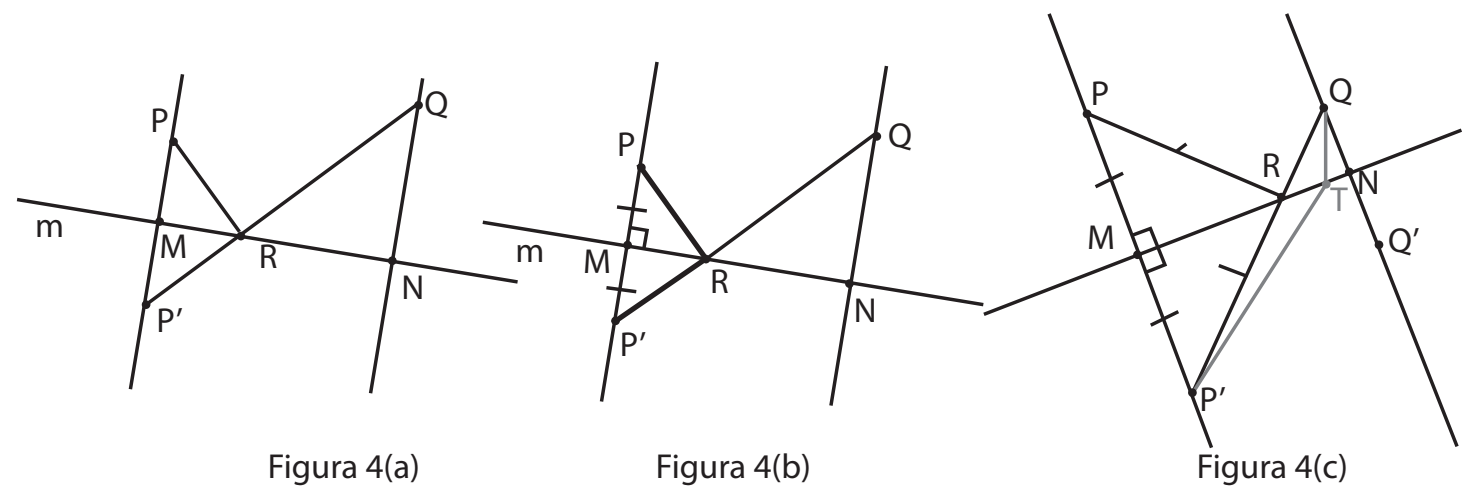

Figura 4(a)

Figura 4(b)

Figura 4(c) 
Uso de la función de arrastre para generar experiencias de aprendizaje de la demostración en geometría Leonor Camargo | Patricia Perry | Carmen Samper | Óscar Molina | Armando Echeverry

\begin{tabular}{|c|c|}
\hline Darío: & $\begin{array}{l}\text { Entonces pues, como está el segmento } P^{\prime} Q \text { y los puntos }\left[P^{\prime} \text { y } Q\right] \text { están en } \\
\text { dos semiplanos distintos entonces intersecan a la recta } m \text { en } R \text {. }\end{array}$ \\
\hline Profesora: & $\begin{array}{l}\text { Escogió... P y } Q \ldots \text { están en el mismo semiplano; y escogió } P^{\prime} \text { y } Q \text { que } \\
\left.\text { están en semiplanos opuestos... el segmento }\left[P^{\prime} Q\right] \text { corta [la recta } m\right] \text {. }\end{array}$ \\
\hline Darío: & $\begin{array}{l}\text { Entonces esta suma... como este punto }[R] \text { está entre } P^{\prime} \text { y } Q \text {, entonces } \\
\text { puedo decir que } P^{\prime} R \text { más } R Q \text { es igual a } P^{\prime} Q \text { [Escribe: } P^{\prime} R+R Q=P^{\prime} Q ; \text { usa la } \\
\text { definición de interestancia]. Entonces... si cojo otro punto de la recta... }\end{array}$ \\
\hline Profesora: & $\begin{array}{l}\text { Pero primero te tengo una pregunta... en el enunciado [del problema] } \\
\text { decía... era } P R \text { más } R Q \ldots \text { y tú tienes } P^{\prime} \ldots\end{array}$ \\
\hline Darío: & $\begin{array}{l}\text { ¡A nho! es que después se hace que... como tengo un punto }[M] \text { en la } \\
\text { recta y una perpendicular [a } m \text { por } M \text { ] y trazo el segmento } P R \text {, entonces } \\
\text { [los triángulos] } P R M \text { y } P^{\prime} R M \text { van a ser congruentes por criterio lado- } \\
\text { ángulo-lado [Figura } 4(\mathrm{~b})] \text {. }\end{array}$ \\
\hline Profesora: & $\begin{array}{l}\text { [Dirigiéndose al resto de la clase } ¿ \text { De acuerdo? Porque él construyó este } \\
\text { segmento }[M P] \text { congruente a este }[P M] \text { y }[\text { los triángulos] comparten un } \\
\text { lado }[M R] . . . \text { bien. O sea que esta suma }\left[P^{\prime} R+R Q=P^{\prime} Q\right] \text { es exactamente la } \\
\text { que queríamos y ahora sí le doy permiso a Darío de borrar el "prima". }\end{array}$ \\
\hline Darío: & $\begin{array}{l}\left.\text { [Borra el "prima" y deja: } P R+R Q=P^{\prime} Q\right] \text {. Bueno, entonces cojo otro punto } \\
\text { cualquiera en la recta. }\end{array}$ \\
\hline Profesora: & ¿Aquí? [Dibuja un punto T cualquiera] \\
\hline Darío: & $\begin{array}{l}\left.\text { [Traza los segmentos } P^{\prime} T \text { y } T Q \text {, como se ilustra en la Figura } 4(c)\right] \text {. Sí... } \\
\text { entonces ya no se cumpliría la interestancia } Q-T-P^{\prime} \text { [pues } T \text { no está entre } \\
Q \text { y } P \text { ]. Entonces tenemos un triángulo. Entonces, por el criterio de } \\
\text { desigualdad del triangulo tengo que } P^{\prime} T \text { más } T Q \text { es mayor que } P^{\prime} Q \text { o sea } \\
P^{\prime} T \text { más } T Q \text { es mayor que } P R \text { más } R Q \text {. Y eso pasa con cualquier punto que } \\
\text { se tome. }\end{array}$ \\
\hline Profesora: & $\begin{array}{l}\text { O sea [usó] desigualdad triangular... ¿sí?... Bueno... entonces, esa es } \\
\text { la propuesta de ellos [Se refiere a Darío y su compañero de grupo] y ya } \\
\text { sabemos que funciona. }\end{array}$ \\
\hline
\end{tabular}

Una vez Darío demostró la conjetura (vi), la profesora sugirió estudiar las demás conjeturas, comparándolas con esa. Usó la construcción hecha por Darío y su compañero para proyectar la pantalla de Cabri en la pared y, mediante el arrastre, ubicó a $P$ y a $Q$ en la posición sugerida en la conjetura (i), pidiendo a los estudiantes examinar qué sucedía con el punto $R$. Por esta vía identificaron que esa conjetura era un caso "particular" de la conjetura (vi) y por ende, que su justificación era trivial (pues basta usar el teorema de la mínima distancia). Luego retornó a $P$ y $Q$ a la situación inicial, cons- truyó el punto medio del segmento $M N$ (Figura 3 (vi)) y arrastró $Q$ hasta que $P$ y $Q$ quedaran equidistantes de la recta $m$. Al hacerlo, pudieron observar que el punto $R$ coincidía con el punto medio del segmento, por lo que podían afirmar que la conjetura (ii) era otro caso particular de la conjetura (vi) y podía demostrarse por la misma vía o usando congruencia de triángulos.

Para comparar la conjetura 'problemática' (iii) con la conjetura (vi) la profesora determinó, en la construcción hecha por Darío y su compañero, un punto $A$ diferente 
de $R$ sobre la recta $m$, construyó los segmentos $P A$ y $A Q$, hizo el cálculo de la suma de las distancias y adicionalmente tomó las medidas de los ángulos PAM y QAN (ver Figura 5(a)). Después, arrastró al punto $A$ buscando el lugar en el que la suma de las distancias era la mínima; allí la medida de los ángulos era igual y $R$ y $A$ coincidían; esto quería decir que las construcciones producían el mismo punto (Figura 5(b)).

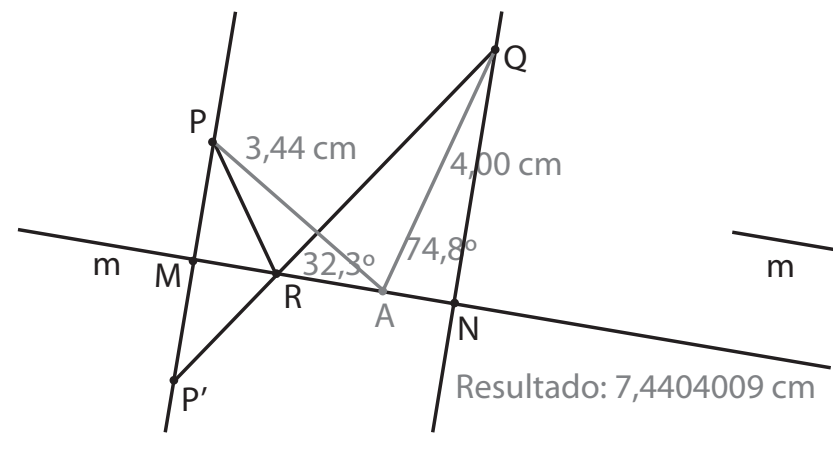

Figura 5(a)

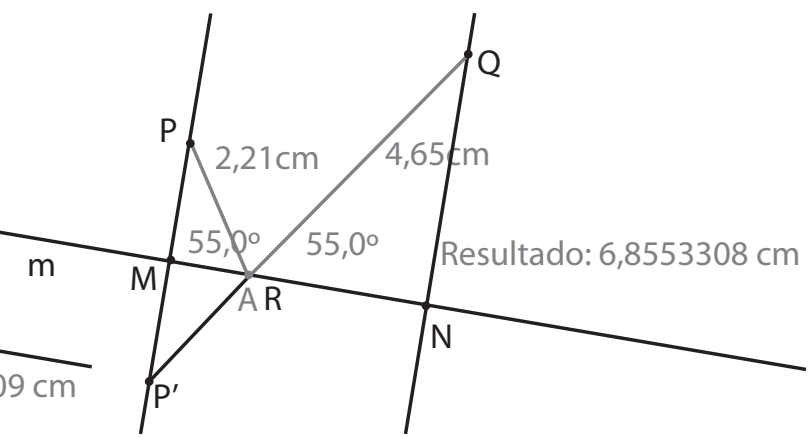

Figura 5(b)
Una vía similar se empleó para verificar que las construcciones sugeridas en las conjeturas (iv) y (v) conducían al mismo punto $R$ sugerido por Darío y su compañero. En síntesis, después de establecer la validez teórica de la conjetura (vi), la actividad de clase se centró en hacer una verificación empírica que llevara a inferir que las conjeturas (iii), (iv) y (v) pueden establecerse como consecuencia de la conjetura (vi), usando la configuración utilizada para su demostración.

A través de la verificación por arrastre se concluyó que las construcciones propuestas en las conjeturas (iii) y (vi) determinaban el mismo punto. El reto estaba en validar teóricamente la conjetura (iii), ya que ésta no sugería propiedades geométricas que pudieran ser justificadas con elementos del sistema axiomático. A raíz del trabajo de comparación de las conjeturas y en un esfuerzo por no descartar ninguna idea de los estudiantes, a la profesora se le ocurrió súbitamente aventurarse a hacer un silogismo para demostrar que el punto $R$, obte- nido mediante la construcción sugerida en la conjetura (iii), tenía las propiedades geométricas del punto $R$, producto de la construcción sugerida en la conjetura (vi), y cómo Darío ya había demostrado que el segundo punto $R$ cumplía la condición pedida en el enunciado del problema, podría concluirse que el primer punto $R$ también cumpliría la condición. Para ello, propuso a los estudiantes partir de la congruencia de los ángulos $\angle 1$ y $\angle 2$ (Figura 6(a)), como única condición "dada" para el punto $R$ — tal cómo lo sugería la conjetura (iii) - y buscar entre todos cómo demostrar que $R$ era colineal a $Q$ y a un punto sobre la perpendicular a $P M$ por $m$ que se encontraba a la misma distancia de $m$ que el punto $P$, tal cómo lo indicaba la conjetura (vi) (Figura 3(vi)).

María sugirió trazar el rayo opuesto al rayo $R Q$ y determinar el punto $S$ de intersección del rayo con la perpendicular $P M$ a $m$; así, explicó María, se garantizaba que $S, R$ y $Q$ eran colineales, pues la definición de "rayos opuestos" así lo exigía (Figura 6(b)). Faltaba mostrar que la 
Uso de la función de arrastre para generar experiencias de aprendizaje de la demostración en geometría Leonor Camargo | Patricia Perry | Carmen Samper | Óscar Molina | Armando Echeverry

distancia SM era igual a la distancia MP. Melisa argumentó que esa equidistancia se tenía puesto que el $\angle 1$ era congruente con el $\angle 3$ (por ser $\angle 3$ opuesto por el vértice a $\angle 2$ y éste ser congruente a $\angle 1$; Figura $6(c)$ ), los ángulos
PMR y SMR eran rectos y el lado MR era común; esto quería decir que los triángulos $P M R$ y $S M R$ eran congruentes y por lo tanto $P M=M S$. El punto $S$ correspondía entonces al punto $P^{\prime}$ de la conjetura (vi).

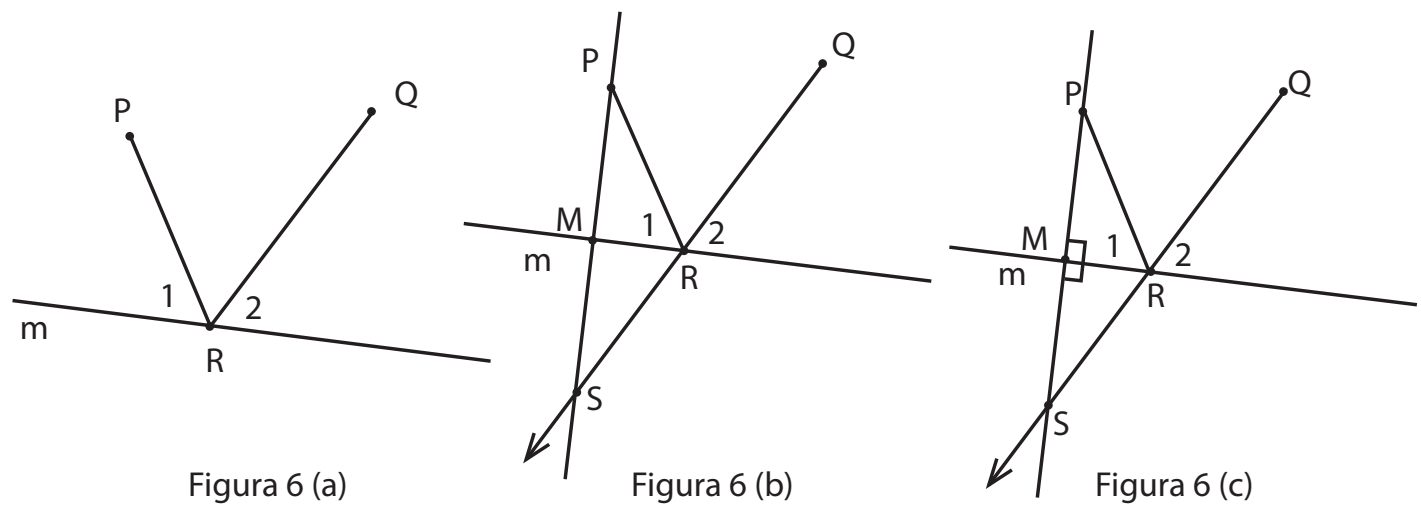

De esta forma demostraron que si $\angle 1$ y $\angle 2$ eran congruentes entonces $R$ era colineal a los puntos $S$ y $Q$ y además $P M=M S$; y como ya habían demostrado que si $R$ era colineal a los puntos $S$ y $Q$ y $P M=M S$ entonces $P R+R Q$ era la suma mínima, pudieron concluir que si $\angle 1$ y $\angle 2$ eran congruentes, entonces $P R+$ $Q R$ era la suma mínima. Por un camino similar, se validó la conjetura 'problemática' (iv) y quedó pendiente hacer algo similar con la conjetura (v), pues en el tiempo restante de la clase no hubo oportunidad para detenerse a analizar esta conjetura.

\section{Análisis de la experiencia a manera de conclusión}

Desde nuestro punto de vista, la experiencia particular que narramos en la sección anterior ilustra la dinámica de una clase orientada bajo la perspectiva del aprendizaje y no de la enseñanza. A pesar de que hay una planeación por parte de la profesora, en la que se previó un problema para involucrar a los estudiantes en la producción de una conjetura y la demostración de ésta usando enunciados del sistema axiomático, ella no descartó las conjeturas que se salían de sus previsiones. Efectivamente, suponía que los estudiantes propondrían conjeturas como la formulada por Darío y su compañero y tenía claro cómo apoyar a Darío cuando éste se animó a hacer la demostración. Además, tenía previsto presentar públicamente las conjeturas "problemáticas" para analizar en qué radicaba el hecho de ser problemáticas, es decir, en el sentido de no sugerir una vía geométrica constructiva del punto $R$. Sin embargo, al momento de relacionar la conjetura (vi) (Figura 3) con las conjeturas (i) y (ii) aprovechando el arrastre para modificar las posiciones de los puntos $P$ y $Q$, tuvo la idea de usar también el arrastre para poner en evidencia que la conjetura (iii) proponían el mismo punto $R$ sugerido en la conjetura (vi) como solución y se animó a intentar una validación con la ayuda de los estudiantes. Así, logró una participación significativa de los estudiantes que formularon la conjetura 
(iii) pues ésta se constituyó en idea discutida y trabajada en la clase (en lugar de descartada) y de quienes le sugirieron cómo avanzar en la producción del silogismo pues ellos dieron las ideas claves de la demostración.

Profesora y estudiantes vivieron una experiencia colectiva de construcción de conocimiento mediada por el uso del arrastre del programa de geometría dinámica. La profesora impulsó el uso de Cabri para dar significado a la conjetura (iii) en el marco del sistema axiomático que estaban construyendo y con ello hizo un uso original de la función de arrastre de Cabri. Como representante de la cultura matemática de referencia y atenta a la intención didáctica de dar sentido a las ideas de los estudiantes para que ellos fueran realmente partícipes de la construcción de conocimiento logró dar un viraje a la planeación de la clase y aprovechó a Cabri para estimular la evolución de significados compartidos hacia la validación de la conjetura (iii) de acuerdo a las normas establecidas en la clase sobre cómo hacerlo. Por su parte, los estudiantes se responsabilizaron de sacar adelante una idea, con la certeza de que no se trataba de mostrar a la profesora lo que ellos sabían sino de contribuir genuinamente con la búsqueda de una vía de demostración de una conjetura. En ese sentido participaron legítimamente en la producción de conocimiento.

Como lo señala Mariotti (2009) el éxito de la experiencia particular reportada en las líneas anteriores se debe en gran medida al papel de la profesora en términos de prestar atención y controlar la dialéctica surgida entre la evolución de los significados compartidos en la clase y la orientación de éstos de manera consistente con las metas didácticas. Asumir una perspectiva sociocultural del aprendizaje de las matemáticas contribuye a asegurar el equilibro dialéctico mencionado al ver la matemática como un producto cultural y también como un objetivo de enseñanza y aprendizaje. En particular, explotar los recursos de mediación que se tienen a disposición para involucrar a los estudiantes en una participación legítima en la producción de conocimiento, es una vía que parece promisoria en esta empresa.

\section{Referencias bibliograficas}

Alibert, D. y Thomas, M. (1991). Research on Mathematical proof. En D. Tall (Ed.), Advanced mathematical thinking. (pp. 215-229). Dordrecht: Kluwer Academic Publishers.

Arzarello, F., Olivero, F., Paola, D. y Robutti, O. (2002). A cognitive analysis of dragging practices in Cabri environments. ZDM. 34(3), 66-72.

Godino, J. y Recio, A. (2001). Significados institucionales de la demostración. Implicaciones para la Educación Matemática. Enseñanza de las Ciencias. 19 (3), 405-414.

Hanna, G. (2000). Proof, explanation and exploration: an overview. Educational Studies in Mathematics. 44, 5-23.

Herbst, P.G. (2002). Establishing a custom of proving in American school geometry: Evolution of the two- column proof in the early twentieth century. Educational Studies in Mathematics. 49, 283-312.

Hoyles, C. (1997). The curricular shaping of students' approaches. For the learning of Mathematics. 17(1), 7-16.

Mariotti, M.A. (2006). Proof and proving in mathematics education. En A. Gutiérrez y P. Boero (Eds.), Handbook of research on the psychology of mathematics education ( $\mathrm{pp}$. 173-204). Rotterdam: Sense Publishers. 
Uso de la función de arrastre para generar experiencias de aprendizaje de la demostración en geometría Leonor Camargo | Patricia Perry | Carmen Samper | Óscar Molina | Armando Echeverry

Mariotti, M.A. (2009). Artifacts and signs after a Vygotskian perspective: The role of the teacher. ZDM Mathematics Education. 41, 427-440.

Olivero, F. (1999). Cabri-Géomètre as a mediator in the process of transition to proofs in open geometric situations: An exploratory study. En W. Maull y J. Sharp (Eds.), Proceedings of the Fourth International Conference on Technology in Mathematics Teaching (CD-ROM). University of Plymouth, Plymouth.
Radford, L. (1994). La enseñanza de la demostración: aspectos teóricos y prácticos. Educación Matemática. 6(3), 21-36.

Wenger, E. (1998). Comunidades de práctica. Aprendizaje, significado e identidad. Barcelona: Paidós. 